\title{
PP07
}

\section{HDR IMAGING FOR LUMINANCE AND MELANOPIC RADIANCE: CAMERAS AND SPECTRAL POWER DISTRIBUTIONSRACT TITLE}

\author{
Thijs Kruisselbrink et al.
}

DOI 10.25039/x47.2020.PP07

\section{Paper accepted for the $5^{\text {th }}$ CIE Symposium on Colour and Visual Appearance}

The paper was selected by the International Scientific Committee (ISC) for presentation at the 5th CIE Symposium on Colour and Visual Appearance, Hong Kong, CN, April 21-22, 2020, which, due to the corona pandemic, could not take place. The paper has not been peer-reviewed by CIE.

\section{(C) CIE 2020}

All rights reserved. Unless otherwise specified, no part of this publication may be reproduced or utilized in any form or by any means, electronic or mechanical, including photocopying and microfilm, without permission in writing from CIE Central Bureau at the address below. Any mention of organizations or products does not imply endorsement by the CIE.

This paper is made available open access for individual use. However, in all other cases all rights are reserved unless explicit permission is sought from and given by the CIE.

CIE Central Bureau

Babenbergerstrasse 9

A-1010 Vienna

Austria

Tel.: +4317143187

e-mail: ciecb@cie.co.at

www.cie.co.at 


\title{
PP07 \\ HDR IMAGING FOR LUMINANCE AND MELANOPIC RADIANCE: CAMERAS AND SPECTRAL POWER DISTRIBUTIONS
}

\author{
Kruisselbrink, T.W., Dangol, R., van Loenen, E.J. \\ ${ }^{1}$ Eindhoven University of Technology, Eindhoven, THE NETHERLANDS \\ 2 Intelligent Lighting Institute, Eindhoven, THE NETHERLANDS \\ t.w.kruisselbrink@tue.nl
}

\begin{abstract}
The luminance, measured using HDR imaging, is relevant to achieve quality lighting. However, the accuracy and applicability of such systems are not warranted. In this study, the capabilities of six different cameras to measure the luminance and melanopic radiance were assessed, based on simulations, relative to 205 spectral power distributions. Moreover, the luminance was determined based on the conventional model and an alternative model aiming to limit the spectral mismatch. The spectral responsivity of the cameras as well as the correlated colour temperature and the full spectrum index showed to be affecting the measurement performance. Large gains in performance were achieved if the luminance calculation was optimized by limiting the spectral mismatch $(10,3 \%$ to $2,9 \%)$. Moreover, for melanopic radiance measurements, large differences in measurement errors occurred between cameras $(20,0 \%$ to $1,9 \%)$. As a result, the camera and luminance calculation model should be chosen carefully.
\end{abstract}

Keywords: Luminance; Melanopic Radiance; Spectral Mismatch; Camera

\section{Introduction}

The luminance distribution, relevant for high quality lighting (Gentile et al., 2016; Kruisselbrink et al., 2018; Van Den Wymelenberg, 2012), can be measured using camera-based systems, such as high-end, commercially available, luminance cameras but also using off-the-shelf cameras (Inanici, 2006; Kruisselbrink et al., 2017). These systems generally use High Dynamic Range (HDR) imaging, where sequential exposure bracketing is applied to capture the dynamic range of the real world (Reinhard et al., 2006). Subsequently, the luminance can be determined by combining the Red $(R)$, Green $(G)$ and Blue $(B)$ tristimuli values of each pixel of the HDR image.

Nevertheless, the accuracy and applicability of these camera systems can still be improved. The luminance is generally calculated using fixed weighting factors for the $R, G$, and $B$ tristimuli originating from the translation of the $S R G B$ to $X Y Z$ colour space, irrespective of camera spectral responsivity and scene illuminant. It is believed that this model which uses these fixed weighting factors has an negative impact on the measurement accuracy as they can introduce significant spectral mismatches due to its assumptions (Cauwerts et al., 2019; Kruisselbrink et al., 2019). First, the Spectral Power Distribution (SPD) is generally not identical to the CIE Standard Illuminant D65. Cai performed identical measurements under different types of SPDs and found significant differences in performance (Cai, 2011), indicating that the SPD has an influence on the accuracy. Second, the camera spectral responsivity does not necessarily align with the $s R G B$ responsivity. For instance, Wu et al. indicated that the spectral responsivity of the camera can have severe disparity with the RGB colour space as manufacturers aim to achieve compelling colours (Wu et al., 2019). As a result, different cameras, with different spectral responsivities, might have varying capabilities in terms of luminance distribution measurements.

Moreover, the applicability of luminance cameras can be extended by measuring other spectral sensitivities such as a-opic radiances that are found to be relevant for the Non-Image Forming (NIF) effects of light (CIE, 2018). Especially, the melanopic radiance, impacting the intrinsically photosensitive retinal ganglion cells (ipRGCs) can be considered important as this results in biological and behavioural effects of light (Lucas et al., 2014). However, the integration process 
of the different $\alpha$-opic radiances is not understood completely yet. Therefore, it is recommended to provide all $\alpha$-opic quantities $(n=5)$. This system is currently also adopted by the CIE (CIE, 2018).

The objective of this study is to simulate the capabilities of six different cameras in relation to luminance measurements using HDR imaging. Moreover, we explore to what extent the accuracy of each camera can be improved by using an alternative model, incorporating the spectral responsivity and the spectral power distribution (SPD), to determine the luminance. Additionally, the alternative model was also applied to explore the feasibility of measuring aopic radiances using these camera systems, which might make such systems very suited for human centric lighting applications. In this study only the melanopic radiance was simulated using the six different cameras as a proof of principle.

\section{Method}

Six commercially available cameras were selected, ranging from high-end DSLRs to simple smartphone cameras with, visually, significantly different spectral responsivities. Their spectral responsivities were selected from $400 \mathrm{~nm}$ to $720 \mathrm{~nm}$ with steps of $10 \mathrm{~nm}$. The green and blue channels of the cameras are illustrated in Figure 1. The spectral responsivities originate from a database by Jiang et al. (2013). The spectral responsivities of HDR images was considered identical to the camera's raw spectral responsivity (Lenseigne et al., 2013). Additionally, 205 SPDs, as illustrated in Figure 2, of light sources that are commercially available were collected, containing LEDs (117), fluorescents (35), incandescent (17), halogens (31), metal halides (4) and sodium pressure lamps (1) from $300 \mathrm{~nm}$ to $900 \mathrm{~nm}$ with steps of $0,5 \mathrm{~nm}$ originating from the LSPDD database by Roby and Aubé (2012).

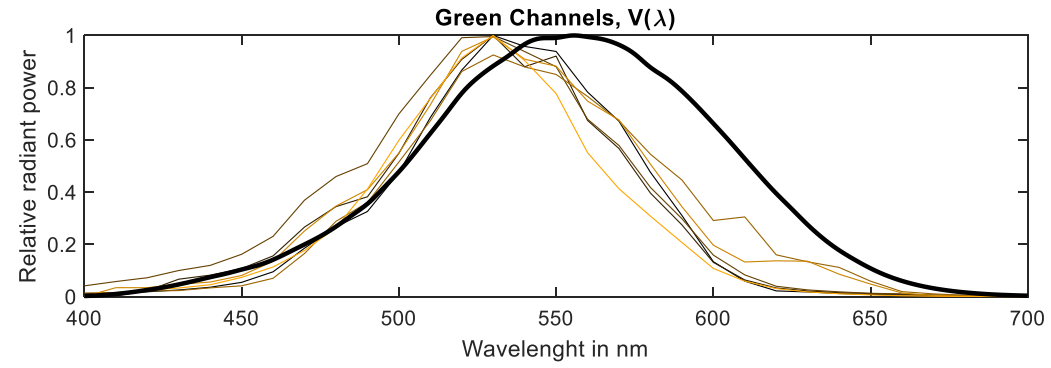

Blue Channels, $\mathbf{S}_{\text {mel }}(\lambda)$

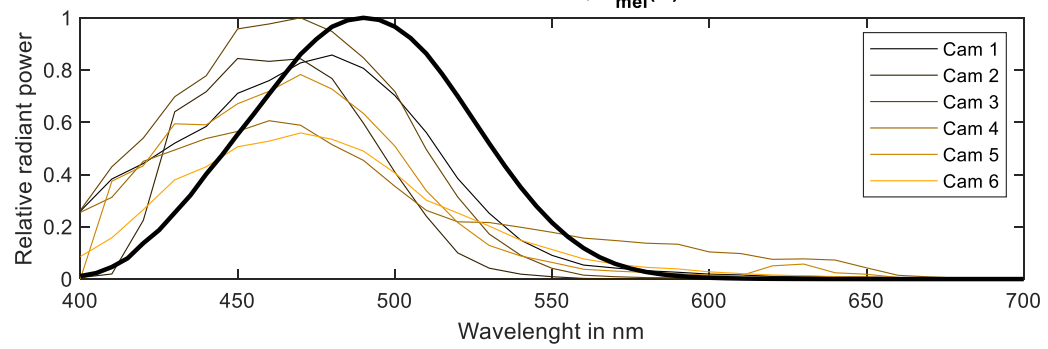

Figure 1 - The spectral responsivities of the green and blue channels of the 6 cameras. Additionally, the luminous efficiency curve $V(\lambda)$ and the melanopic sensitivity $S_{\text {mel }}(\lambda)$ are illustrated in black.

The luminance, considering one single pixel, was calculated based on simulations, using MATLAB r2017a, for all 205 SPDs using the spectral responsivities of the six cameras and two distinct luminance models that were proposed by Kruisselbrink et al. (Kruisselbrink et al., 2019). These models determine the weighting factors of the $R, G$, and $B$ tristimuli according to the conventional and a spectral mismatch indicator $\left(f_{1}^{\prime}\right)$ optimization. The latter method is a camera and SPD dependent optimization incorporating the effect of the camera's spectral responsivity as well as the SPD of the light source.

In the conventional method, the luminance $(L)$ was calculated using a linear combination of the $R, G$, and $B$ coefficients using fixed weighting factors in order to approximate the luminous 
efficiency curve $V(\lambda)$. The linear combination was based on the transformation of the sRGB colour space to the XYZ colour space applying "reference primaries, CIE standard illuminant D65, and standard CIE Colorimetric Observer with $2^{\circ}$ field of view” (Inanici, 2006). The luminance, according to the conventional method, was calculated according to Equation (1).

$$
\mathrm{L}=k \cdot(0.2125 \cdot R+0.7154 \cdot G+0.0721 \cdot B)
$$

where
$L \quad$ is the luminance;
$k \quad$ is the photometric calibration factor;
$R \quad$ is the red channel;
$G \quad$ is the green channel;
$B \quad$ is the blue channel.

The alternative luminance model was based on the General $V(\lambda)$ Mismatch Index $f_{1}^{\prime}(\mathrm{CIE}, 2014)$, generally used to indicate the spectral properties for general light measurements. The metric was applied because a single pixel of a luminance camera can be considered a photometer. The inputs for this metric were the $V(\lambda)$ and the spectral responsivities as illustrated in Figure 1. Moreover, instead of Standard Illuminant D65, the 205 SPDs served as input to determine the most suitable weighting factors for $\mathrm{R}, \mathrm{G}$ and $\mathrm{B}$ for each individual SPD according to Equation (2). The weighting factors that were found were applied in a similar fashion as the conventional method. For an extensive explanation of this model we refer to (Kruisselbrink et al., 2019).

$$
\arg \min f_{1}^{\prime} \text {, subject to: }\left\{\begin{array}{c}
r \in(0,1) \\
g \in(0,1) \\
b \in(0,1) \\
r+g+b=1
\end{array}\right.
$$

where

$f_{1}^{\prime} \quad$ is the General $V(\lambda)$ Mismatch Index;

$r \quad$ is the weighting factor for the red channel;

$g \quad$ is the weighting factor for the green channel;

$b \quad$ is the weighting factor for the blue channel.

The simulations resulted in weighting factors for the $R, G$, and $B$ channel for each individual camera and SPD. The measurement error $\left(\delta_{L}\right)$ between the approximated luminance using the simulated cameras and the actual luminance (perfect $V(\lambda)$ match) was assessed based on the correlated colour temperature (CCT) and the full spectrum index (FSI), which are both onedimensional indicators of the SPD that were expected to have an effect on the luminance calculation performance. 

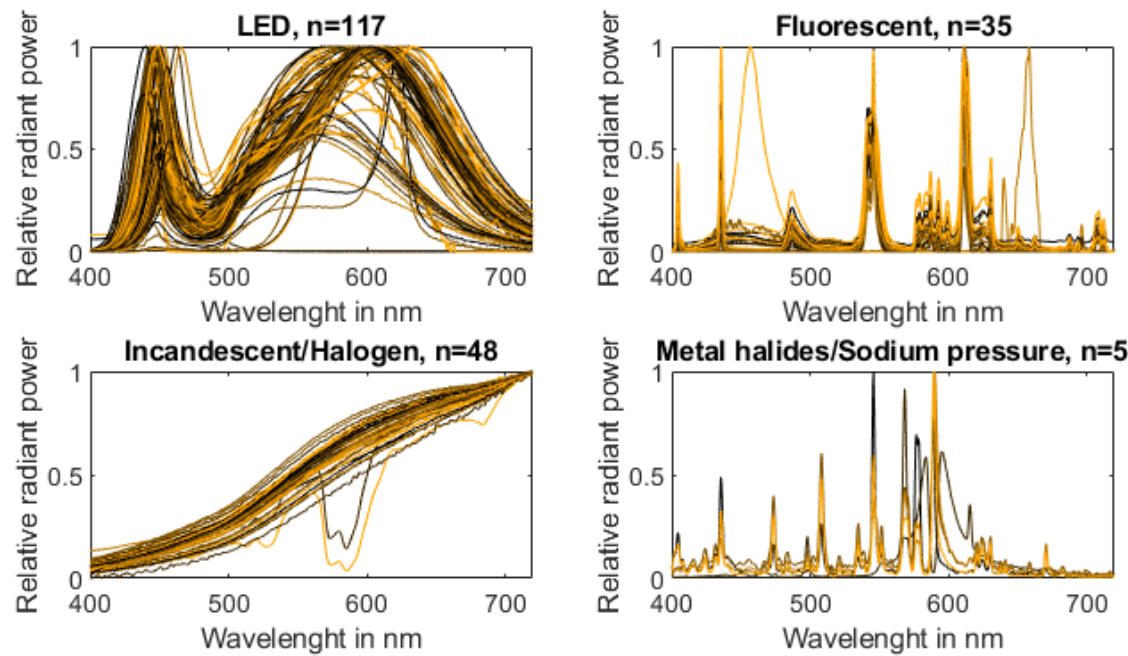

Figure 2 - Implemented spectral power distributions originating from LSPDD database

The CCT, the temperature of a Planckian radiator associated with the chromaticity of the SPD, was calculated according to the method by Hohm and Krochmann (Hohm \& Krochman, 1975). For 7 SPDs the CCT could not be calculated because the distance to the Plackian locus was disproportionate, therefore, these SPDs were not considered for the respective analyses. The FSI (Rea et al., 2005) is a metric that indicates how much a SPD differs from an equal energy spectrum, which was deemed relevant to indicate the continuity of the SPD. The FSI was calculated based on the sum of squared deviations between the cumulative SPD and cumulative equal energy spectrum. A FSI of 0 represents an equal energy spectrum, bigger FSI values are associated with non-continuous, or peaky, SPDs.

Additionally to the luminance, the simulations according to the alternative model were also conducted for the melanopic radiance. The melanopic radiance was determined by replacing the $V(\lambda)$ by the $S_{m e l}(\lambda)$ as illustrated in Figure 1 . The conventional method was disregarded as this was not applicable for the melanopic radiance.

\section{Results}

In this section the simulation results according to the conventional model and the alternative, spectral mismatch based, model for luminance and for melanopic radiance measurements are displayed.

\subsection{Conventional method}

The conventional model to calculate the luminance using cameras introduced large deviations as is illustrated in Table 1 . Spectral mismatches up to $46 \%$ were found, which can be considered very large as the lowest DIN classification for luminance meters is below $5 \%$ (DIN, 2017). Moreover, large differences were found for the measurement error $\left(\delta_{L}\right)$, both the mean as the standard deviation exhibit large differences. High spectral mismatches align with high average (and standard deviation) measurement errors. Based on the results, it is likely that the spectral responsivity of camera 4 has the most similarities with the sRGB responsivity as it performs relatively well.

Table 1 - Average spectral mismatch and average, non-absolute, luminance measurement error for camera 1 to 6 according to the conventional model. The standard deviations are illustrated between brackets.

\begin{tabular}{|l|l|l|l|l|l|l|}
\hline & Cam 1 & Cam 2 & Cam 3 & Cam 4 & Cam 5 & Cam 6 \\
\hline$f_{1}^{\prime}$ & $40,8 \%(4,1 \%)$ & $39,8 \%(2,8 \%)$ & $46,5 \%(3,2 \%)$ & $17,0 \%(1,2 \%)$ & $26.9 \%(1,1 \%)$ & $42,2 \%(4,8 \%)$ \\
\hline$\delta_{L}$ & $-12,7 \%(8,0 \%)$ & $-13,0 \%(11,1 \%)$ & $-16,8 \%(14,1 \%)$ & $-3,6 \%(4,0 \%)$ & $-7,5 \%(6,6 \%)$ & $-13,7 \%(8,4 \%)$ \\
\hline
\end{tabular}


Figure 3 illustrates the absolute measurement error, as indicated in Table 1, relative to the CCT. In general, all cameras show a similar trend where high inaccuracies were introduced for low CCTs. The error decreases towards a CCT of 6500K. This effect was mainly visible for cameras with high spectral mismatches, for cameras with relatively low spectral mismatches the dependency on the CCT, or SPD, was limited. The error decreased towards a CCT of $6500 \mathrm{~K}$ because this is the CCT of the standard illuminant applied in the conventional method. It is expected that for CCTs higher than 6500K the errors will increase again. This indicates that there is a dependency on the SPD, indicated using the CCT (Figure 3), when the conventional method is applied to calculate the luminance.

A similar analysis was conducted using FSI as an indicator for the SPD (Figure 4), instead of the CCT. Again, a clear trend was found, showing a dependency on the SPD. Figure 4 illustrates that using the conventional method to calculate the luminance was more accurate for SPDs that have a full spectrum, such as an incandescent. On the other hand, it illustrates that it has more difficulties to measure SPDs that contains peaks such as fluorescent light sources. In contrast to

Figure 3 , this effect was also clearly visible for the cameras with a relatively low spectral mismatch, in these cases only the magnitude of the errors was lower compared to the others.
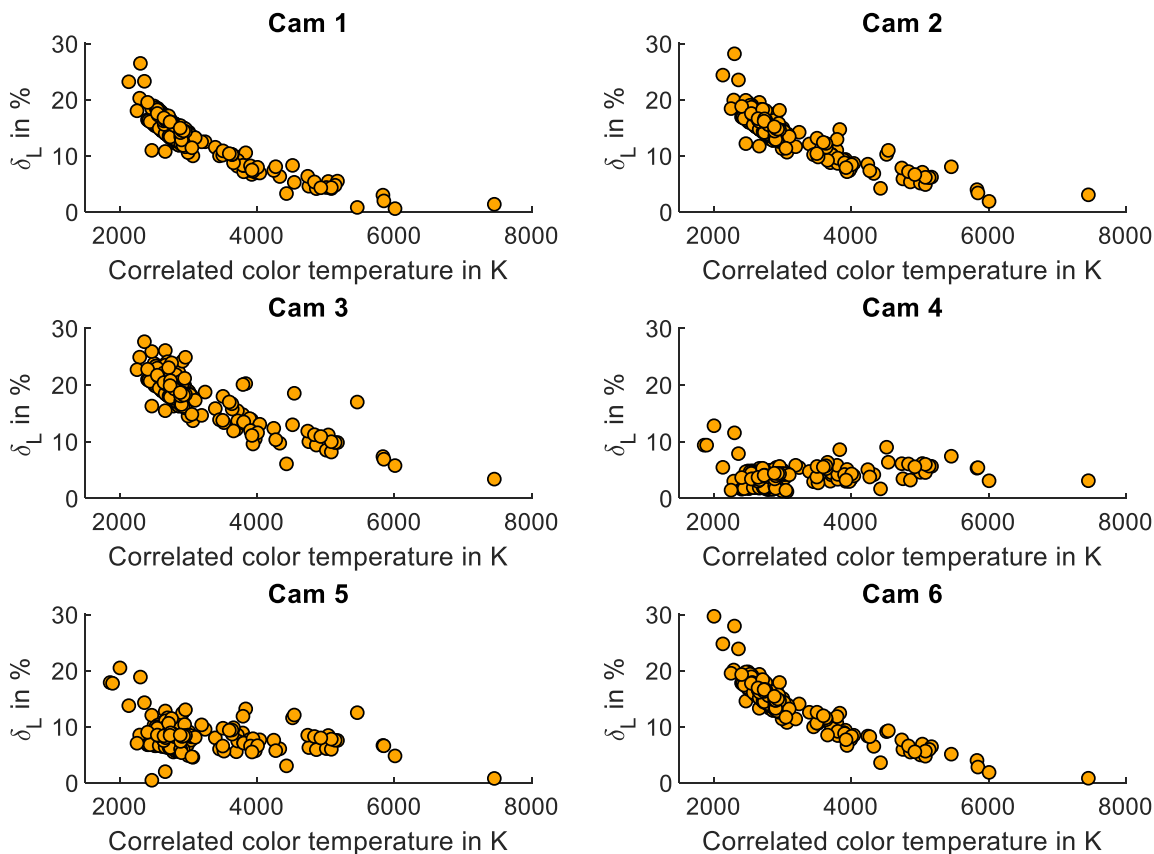

\section{Figure 3 - Relation between the CCT and the absolute measurement error of the luminance} cameras according to the conventional method 

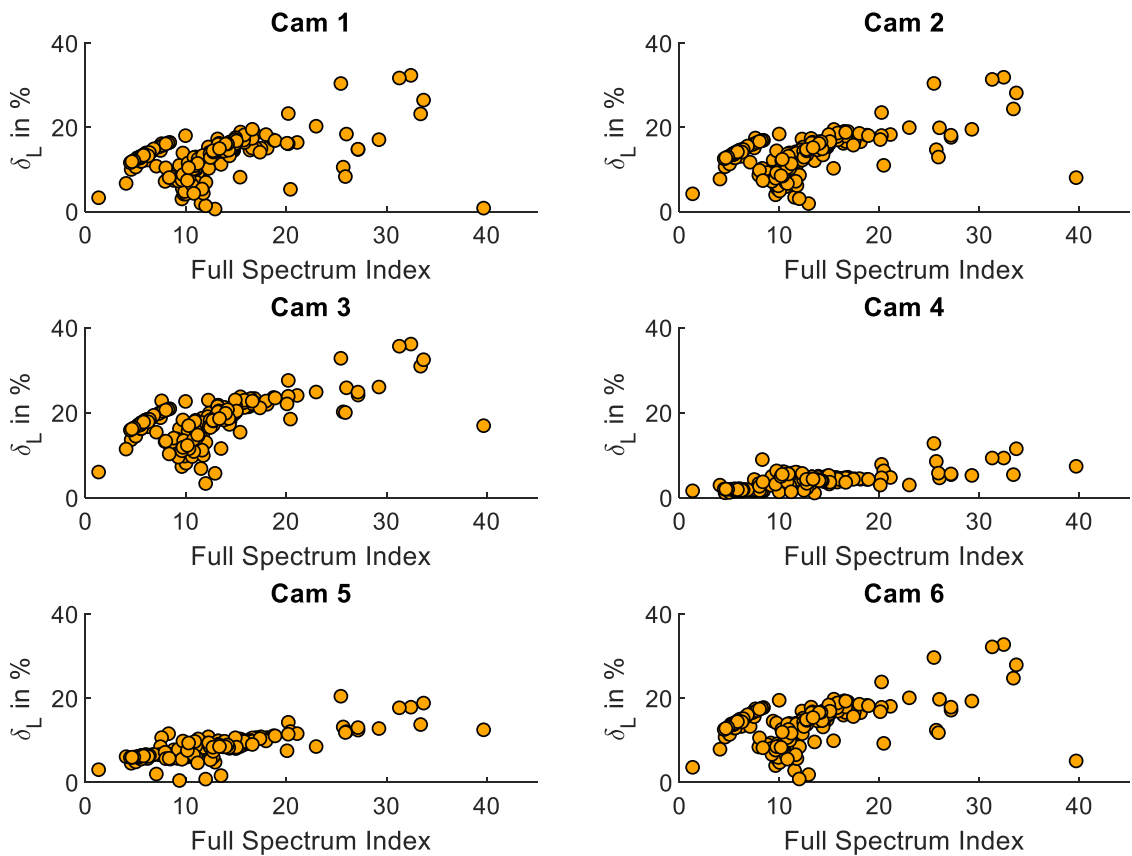

Figure 4 - Relation between the FSI and the absolute measurement error of the luminance cameras according to the conventional method.

\subsection{Spectral mismatch optimization}

Based on the optimized luminance calculation, according to the alternative model, the spectral mismatches and measurement errors have been reduced drastically, as is shown in Table 2. The maximum spectral mismatch has been reduced to approximately $20 \%$ compared to a spectral mismatch of $46 \%$ for the conventional method. Consequently, the measurement error has been decreased to a maximum of approximately $6 \%$. Especially, for camera 6 the optimization was fruitful. On the other hand, the performance for camera 4 did not show a significant improvement. Moreover, for this camera, the weighting factors for $R, G$, and $B$ channels were relatively similar to the conventional method. For the other cameras, the weighting factors were largely different to the conventional method. First, the blue channel was generally not required as the information of the blue part of the spectrum was captured using the green channel. Moreover, a larger part of the red part of the camera was required because the maximum responsivity of the green channel was generally below $550 \mathrm{~nm}$, which requires the red channel to compensate.

Table 2 - Average $r, g, b$ weighting factors, spectral mismatches and, non-absolute, luminance measurement errors for camera 1 to 6 according to alternative model. The standard deviations are illustrated between brackets.

\begin{tabular}{|l|l|l|l|l|l|l|}
\hline & Cam 1 & Cam 2 & Cam 3 & Cam 4 & Cam 5 & Cam 6 \\
\hline $\mathrm{r}$ & $0,52(0,02)$ & $0,42(0,02)$ & $0,46(0,02)$ & $0.21(0,01)$ & $0,28(0,01)$ & $0,51(0,01)$ \\
\hline $\mathrm{g}$ & $0,48(0,02)$ & $0,58(0,02)$ & $0,54(0,02)$ & $0,76(0,04)$ & $0,72(0,01)$ & $0,49(0,01)$ \\
\hline $\mathrm{b}$ & $0,00(0,00)$ & $0,00(0,00)$ & $0,00(0,00)$ & $0,02(0,00)$ & $0,00(0,00)$ & $0,00(0,00)$ \\
\hline$f_{1}^{\prime}$ & $17,7 \%(1,0 \%)$ & $19,8 \%(0,8 \%)$ & $20,8 \%(1,2 \%)$ & $16,0 \%(0,3 \%)$ & $20,9 \%(0,3 \%)$ & $11,0 \%(0,8 \%)$ \\
\hline$\delta_{L}$ & $-1,8 \%(2,9 \%)$ & $-4,5 \%(3,7 \%)$ & $-5,6 \%(4,2 \%)$ & $-2,3 \%(1,8 \%)$ & $-2,8 \%(1,1 \%)$ & $-1,6 \%(2,3 \%)$ \\
\hline
\end{tabular}



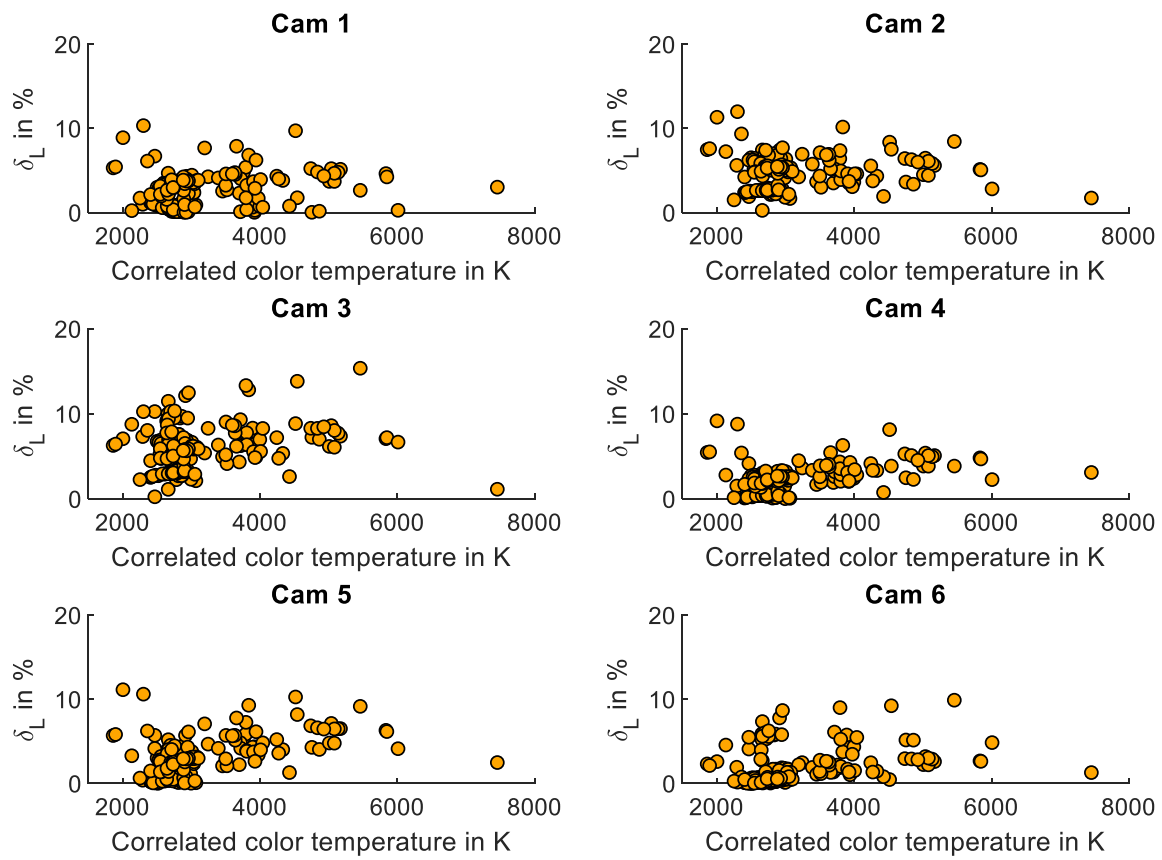

\section{Figure 5 - Relation between the CCT and the absolute measurement error of the luminance cameras according to the alternative model.}

Due to the optimization for each individual SPD the dependency on the CCT was almost nonexisting. Figure 5 illustrates that no clear pattern was occurring between the CCT and the measurement error. In contrast to the conventional method, the maximum absolute error was generally reduced to approximately $10 \%$ compared to a maximum error of $>30 \%$ for the conventional method.

Figure 6 shows that, in contrast to the CCT, the optimization remained dependent on the FSI. The performance for full spectrum SPDs was still higher than for SPDs with peaks. Apparently, continuous SPDs were easier to match using only three channels of the cameras' responsivity. For peaky SPDs the specific wavelengths might not be present in the cameras' responsivity. Again, for high performing cameras, the dependency on the FSI decreases. A hypothetical camera with a perfect spectral match will show no dependency to the FSI or any performance indicator. 

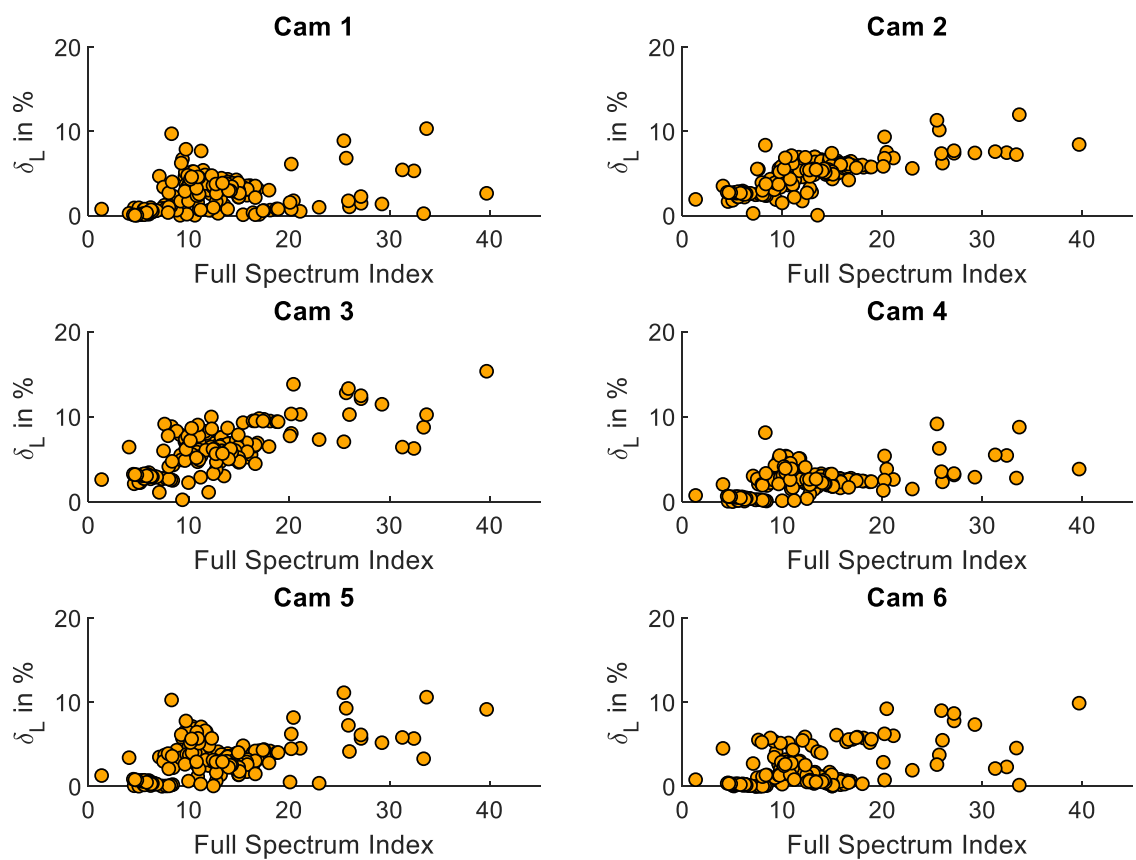

Figure 6 - Relation between the FSI and the absolute measurement error of the luminance cameras according to the alternative model.

\subsection{Melanopic Radiance}

In the analysis, for the melanopic radiance, one single SPD was discarded as it contained one single peak at $650 \mathrm{~nm}$, which was outside the melanopic sensitivity. As expected, the accuracy of the optimized luminance (Section 3.2) was not achieved. Table 3 gives an indication of the measurement capabilities of the cameras for the melanopic radiance. Cameras are, after all, developed for measurements that match our visual experience. Nevertheless, for some cameras the performance was better than for the conventional luminance measurements, although the variance between cameras was quite high. It shows that the capabilities were largely dependent on the spectral responsivity of the camera, for instance, camera 4 performed well for the conventional luminance measurement, but was not able to accurately measure the melanopic radiance. Similar to the optimized luminance, the melanopic radiance was generally measured with only two channels, in this case the red channel was not required. The blue channel was highly normative as this aligns relatively well with the melanopic sensitivity (Figure 1).

Figure 7 illustrates that for cameras that have large measurement errors $\left(\delta_{S}\right)$, there was some dependency to the CCT. Difficulties arise when the melanopic radiance was measured for SPDs with a low CCT. This error was introduced by the misalignment between the high amount of energy for longer wavelengths and the melanopic sensitivity for the lower wavelengths. The resulting measurement errors can be very high (>100\% for camera 4, cropped out of Figure 7 for readability) but for others it was reasonable.

Again,

Figure 8 illustrates an almost linear dependency on the FSI, which is much clearer for the low performing cameras. So, the melanopic radiance is more accurately measured for full spectrums, similar to the findings in Section 3.2. 
Table 3 - Average r,g,b weighting factors, spectral mismatches and, non-absolute, measurement errors for camera 1 to 6 for melanopic radiance measurements $\left(\delta_{s}\right)$. The standard deviations are illustrated between brackets.

\begin{tabular}{|l|l|l|l|l|l|l|}
\hline & Cam 1 & Cam 2 & Cam 3 & Cam 4 & Cam 5 & Cam 6 \\
\hline $\mathrm{r}$ & $0,00(0,00)$ & $0,00(0,00)$ & $0,00(0,00)$ & $0,00(0,00)$ & $0,00(0,00)$ & $0,00(0,00)$ \\
\hline $\mathrm{g}$ & $0,12(0,02)$ & $0,29(0,03)$ & $0,28(0,03)$ & $0,06(0,03)$ & $0,16(0,03)$ & $0,11(0,03)$ \\
\hline $\mathrm{b}$ & $0,88(0,02)$ & $0,71(0,03)$ & $0,72(0,03)$ & $0,94(0,03)$ & $0,84(0,03)$ & $0,89(0,03)$ \\
\hline$f_{1}^{\prime}$ & $33,6 \%(0,8 \%)$ & $39,5 \%(1,0 \%)$ & $37,1 \%(1,0 \%)$ & $62,4 \%(2,0 \%)$ & $44,4 \%(1,0 \%)$ & $37,0 \%(1,7 \%)$ \\
\hline$\delta_{S}$ & $-3,1 \%(2,7 \%)$ & $11,1 \%(5,7 \%)$ & $1,9 \%(3,7 \%)$ & $20,0 \%(26,9 \%)$ & $4,2 \%(4,2 \%)$ & $6,4 \%(7,1 \%)$ \\
\hline
\end{tabular}
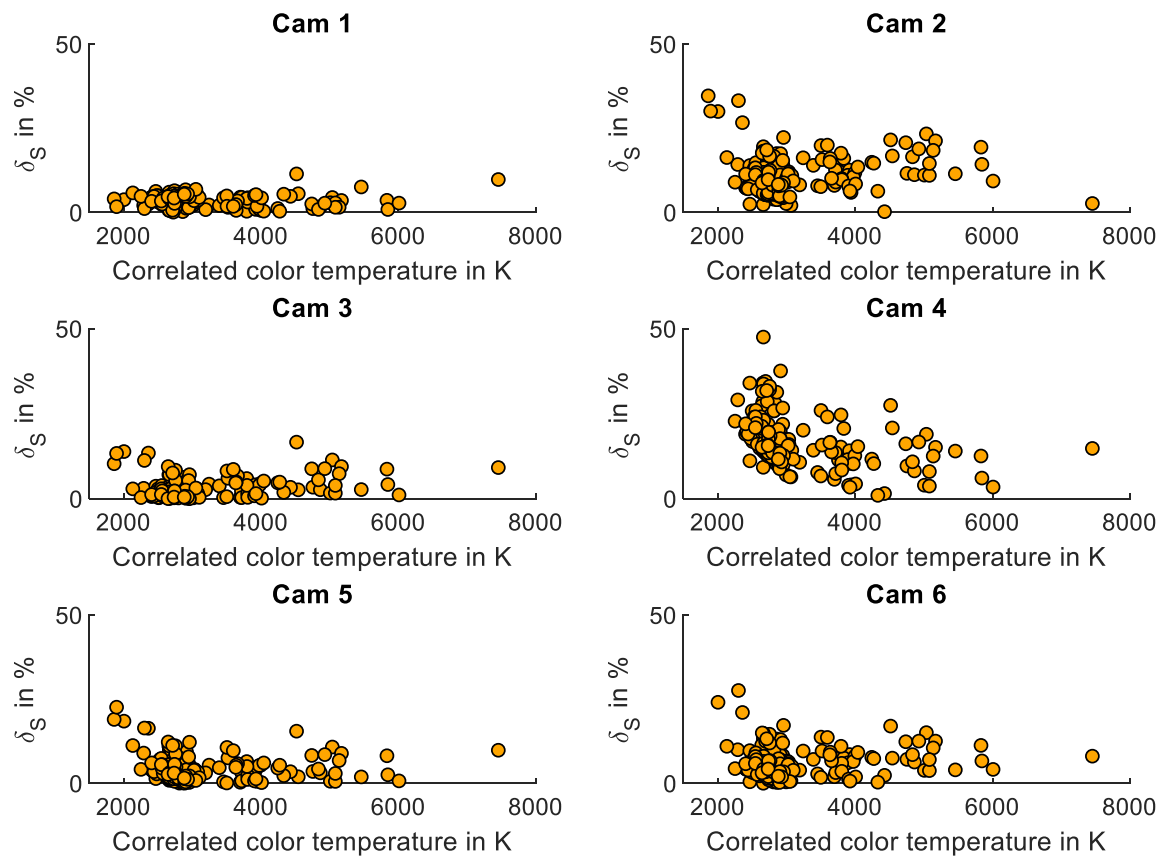
Figure 7 - Relation between the CCT and the absolute measurement error of the melanopic
radiance measurement according to the alternative model

\section{Discussion and Conclusion}

The objective of this study was to assess, based on simulations, the capabilities of six cameras to measure the luminance and melanopic radiance. Besides the conventional model to calculate the luminance, an alternative model, which was based on the General $V(\lambda)$ Mismatch Index, was used to assign the most suitable weighting factors for the $R, G$ and $B$ channels for luminance and melanopic radiance measurements.

The conventional model, to calculate the luminance, introduced significant average luminance measurement errors ranging from approximately $4 \%$ to $17 \%$. In all cases, the alternative model was able to reduce the average measurement errors, ranging from approximately $2 \%$ to $6 \%$, to a large extent. Additionally, some cameras were able to measure the melanopic radiance relatively accurate with average measurement errors below $5 \%$. However, large differences were found between cameras, one camera was not able to provide accurate measurements $(>20 \%)$. 

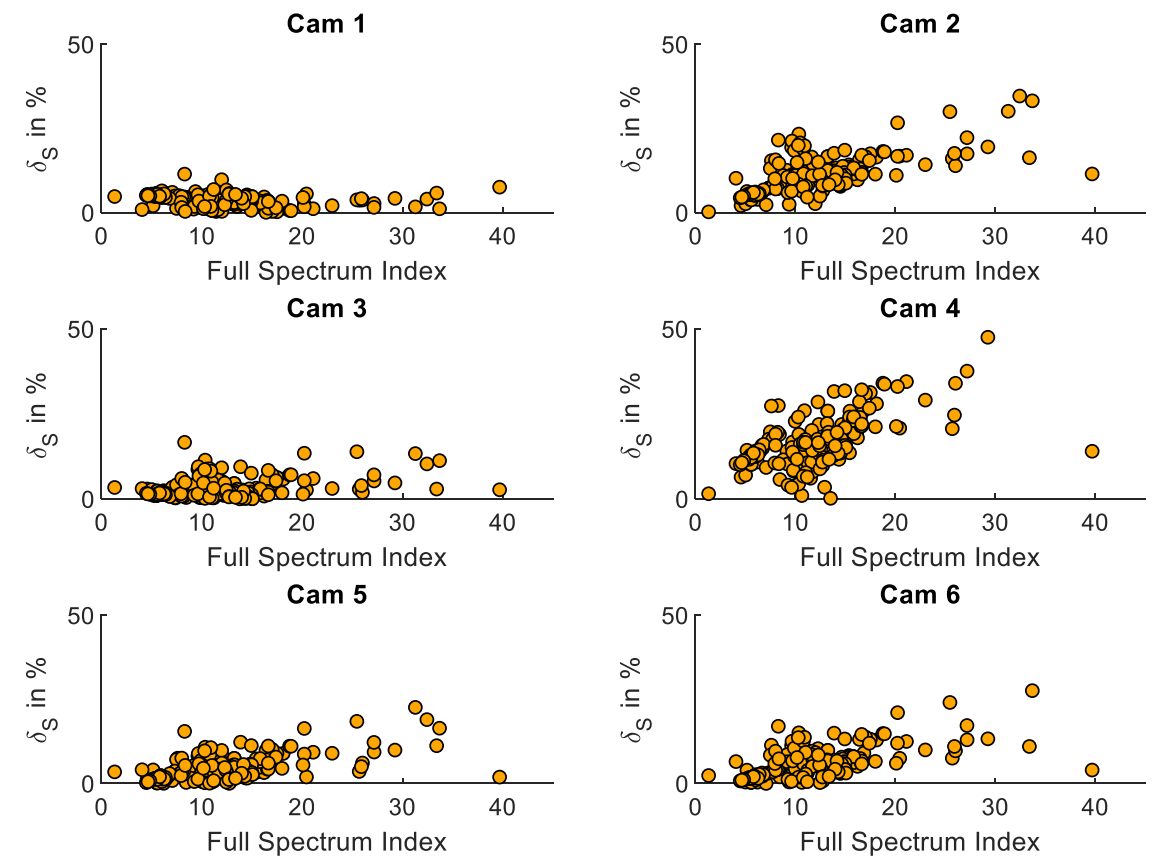

\section{Figure 8 - Relation between the FSI and the absolute measurement error of the melanopic radiance measurement according to the alternative model}

This study indicated that luminance and melanopic radiance measurements are sensitive to the SPD of the light source. Both the CCT and the FSI are proven to influence the performance of the camera systems. The CCT, roughly indicating which wavelengths contained the most energy, affected the performance for measurements with the conventional model and the melanopic radiance measurement. For the conventional method, SPDs very different to standard illuminant D65 had a lower performance because the R, G, and B channels of the camera were combined such that a more bluish $(6500 \mathrm{~K})$ light source was measured accurately. For this reason, the blue channel determined approximately $7 \%$ of the luminance. This effect disappeared for the alternative model because the weighting factors were specifically determined for each individual illuminant, resulting in almost no importance of the blue channel. Nevertheless, the standard deviation for the improved weighting factors was low, indicating that it is fairly safe to use the mean weighting factors instead of SPD dependent weightings (Table 2 and Table 3). For the melanopic radiance, low CCT light sources performed worse as they mainly contained energy in the reddish part of the SPD, while the melanopic sensitivity is sensitive to the blue part. As only three channels, in practice only two, were applied, the blue component could not be extracted exclusively. Consequently, also energy outside the melanopic range was captured, which reduced the performance.

As expected, the FSI showed that continuous SPDs were generally measured more accurately. Especially, very peaky SPDs did not perform well as the sensitivity of the cameras can be very low for these specific wavelengths. For continuous SPDs, the wavelengths with low sensitivity are easily accounted for by the wavelengths with high sensitivity.

Large differences were found between cameras, as their spectral responsivities differ. Hence, their capabilities to capture the luminous or melanopic sensitivities vary. The differences for the optimized luminance measurements were limited as most cameras aim to achieve a visually pleasing image which results in high sensitivity, and overlap between the channels, for the range of the luminous sensitivity $(V(\lambda))$. Using the optimization, the most suitable combination between channels can be found, which differed significantly for the different cameras. The melanopic radiance showed the biggest differences between cameras as their sensitivity was generally low for the relevant wavelengths. Moreover, the blue channels were not aligned as well with the melanopic sensitivity as the green channels were aligned with the luminous sensitivity. 
When a low spectral mismatch is achieved, the dependency on the SPD is low. However, when the spectral mismatch is significant, which is often the case for these camera systems, then the SPD is relevant. For instance, the CCT and FSI of a SPD have a larger influence on low performing cameras as wavelengths outside the region of interest are captured as well.

This study showed that the conventional method to calculate the luminance can introduced significant errors. It is therefore, advised to either pick a suitable camera, which spectral responsivity is suitable, and/or optimize the weighting factors for the $R, G$ and $B$ channels. Consequently, the spectral mismatches can be reduced drastically, reducing the sensitivity to the SPD. Moreover, it was shown that the melanopic radiance can be approximated by such camera systems. However, the applied camera has even higher importance, as some cameras are not able to achieve an acceptable spectral match and differences between weighting factors for different SPDs were limited.

It is recommended to perform physical measurements to validate the results found. In this study, only simulations were conducted, which might not always be a correct impression of reality (Kruisselbrink et al., 2019). For instance, the spectral responsivity of the cameras might be different in practice. Moreover, the imaging pipeline is more complex as illustrated in the applied models. Finally, 205 SPDs were applied that are commercially available. However, in practice the light source will have a mixed character containing daylight as well as an artificial light source.

\section{References}

Cai, H. (2011). Camera Aided Luminance Measurement of the Luminous Surfaces of Different Light Sources. AEI 2011, 272-279. https://doi.org/10.1061/41168(399)33

Cauwerts, C., Jost, S., \& Deroisy, B. (2019). Calibration of high dynamic range images for applied color and lighting research. Journal of the Optical Society of America A, 36(11), C130. https://doi.org/10.1364/JOSAA.36.00C130

CIE. (2014). Characterization of the performance of illuminance meters and luminance meters: Vol. ISO/CIE 19.

CIE. (2018). IE System for Metrology of Optical Radiation for ipRGC-Influenced Responses to Light: Vol. CIE S 026. https://doi.org/10.25039/S026.2018

DIN. (2017). DIN 5032 Photometry - Part 7: Classification of illuminance meters and luminance meters.

Gentile, N., Dubois, M. C., Osterhaus, W. K. E., Stoffer, S., Naves David Amorim, C., GeislerMoroder, D., Jakobiak, R., Amorim, C. N. D., Geisler-Moroder, D., \& Jakobiak, R. (2016). A toolbox to evaluate non-residential lighting and daylighting retrofit in practice. Energy and Buildings, 123, 151-161. https://doi.org/10.1016/j.enbuild.2016.04.026

Hohm, W., \& Krochman, J. (1975). ERMITTLUNG DER AEHNLICHSTEN FARBTEMPERATUR. FARBE, 24(1-6), 91-96.

Inanici, M. N. (2006). Evaluation of high dynamic range photography as a luminance data acquisition system. Lighting Research and Technology, 38(2), 123-136. https://doi.org/10.1191/1365782806li164oa

Jiang, J., Liu, D., Gu, J., \& Susstrunk, S. (2013). What is the space of spectral sensitivity functions for digital color cameras? Proceedings of IEEE Workshop on Applications of Computer Vision, 168-179. https://doi.org/10.1109/WACV.2013.6475015

Kruisselbrink, T. W., Aries, M. B. C., \& Rosemann, A. L. P. (2017). A Practical Device for Measuring the Luminance Distribution. International Journal of Sustainable Lighting, 19(1), 75-90. https://doi.org/10.26607/ijsl.v19i1.76

Kruisselbrink, T. W., Dangol, R., \& Rosemann, A. L. P. (2018). Photometric measurements of lighting quality: An overview. Building and Environment, 138, 42-52. https://doi.org/10.1016/j.buildenv.2018.04.028 
Kruisselbrink, T. W., Dangol, R., Rosemann, A. L. P., \& van Loenen, E. J. (2019). Spectral tuning of luminance cameras: A theoretical model and validation measurements. Lighting Research \& Technology. https://doi.org/10.1177/1477153519880231

Lenseigne, B., Jacobs, V. A., Withouck, M., Hanselaer, P., \& Jonker, P. P. (2013). Color sensitivity of the multi-exposure HDR imaging process. Advanced Optical Technologies, 2(2), 159-169. https://doi.org/10.1515/aot-2013-0002

Lucas, R. J., Peirson, S. N., Berson, D. M., Brown, T. M., Cooper, H. M., Czeisler, C. A., Figueiro, M. G., Gamlin, P. D., Lockley, S. W., O’Hagan, J. B., Price, L. L. A., Provencio, I., Skene, D. J., \& Brainard, G. C. (2014). Measuring and using light in the melanopsin age. Trends in Neurosciences, 37(1), 1-9. https://doi.org/10.1016/j.tins.2013.10.004

Rea, M. S., Deng, L., \& Wolsey, R. (2005). Full-Spectrum Light Sources. Lighting Answers, $7(5)$.

Reinhard, E., Ward, G., Pattanaik, S., \& Debevec, P. (2006). High Dynamic Range Imaging: Acquisition, Display, and Image-Based Lighting (The Morgan Kaufmann Series in Computer Graphics). Morgan Kaufmann Publishers Inc.

Roby, J., \& Aubé, M. (2012). LSPDD / Light Spectral Power Distribution Database. http://galileo.graphycs.cegepsherbrooke.qc.ca/app/en/lamps

Van Den Wymelenberg, K. G. (2012). Evaluating Human Visual Preference and Performance in an Office Environment Using Luminance-based Metrics [University of Washington]. In University of Washington. https://doi.org/10.1017/CBO9781107415324.004

Wu, Y., Kämpf, J. H., \& Scartezzini, J. L. (2019). Design and validation of a compact embedded photometric device for real-time daylighting computing in office buildings. Building and Environment, 148, 309-322. https://doi.org/10.1016/j.buildenv.2018.11.016 